\title{
L'expérience mystique chez Philon
}

\author{
Par SØREN GIVERSEN
}

L'expérience mystique chez Philon d'Alexandrie qu'est-qu'il implique et comment se fait-il? C'est un problème important pour qui veut étudier la mystique en générale, et naturellement aussi pour qui veut rechercher la religiosité de Philon.

Dans son Legum allegoriae Philon dit $^{1}$ au sujet du voũs (l'intelligence): «Mais il y a une intelligence plus parfaite et mieux purifiée, initiée aux grands mystères, qui connaît la Cause non d'après les êtres du devenir, comme on peut connaître l'objet immobile d'après son ombre; ayant dépassé le devenir elle reçoit une claire apparition de l'inengendré, en sorte que, d'après lui, elle comprend et lui et son ombre; c'est-à-dire la raison et ce monde-ci » (Legum allegoriae III, I0o). Cela s'accorde avec la conception de Philon de la possibilité de connaître Dieu². Selon Philon, il est possible d'arriver à une certaine connaissance de Dieu à l'aide de la raison. Ainsi Philon ne nie pas la possibilité d'arriver à une certaine connaissance de Dieu par la voie de la logique; par un examen approfondi de la vie avec ses nombreuses phénomènes il est possible d'arriver à une telle connaissance, mais selon Philon il n'est pas possible de parvenir à une connaissance complète et adéquate par cette voie ${ }^{3}$ - Cependant il serait en place ici de mentionner que chez Philon il n'est pas question de déprécier la logique; dans un passage Philon appelle expressément le principe logique le meilleur de tout ce qu'il existe:

${ }^{1}$ Je cite les œuvres de Philon conservées en grec de l'édition critique publié par L. Cohn et P. Wendland, Philonis Alexandri Opera quae supersunt, I-7, Berlin I896r930 (editio major), et les œuvres conservées en arménien de l'édition publié par J. B. Aucher, Philonis Fudaei Paralipomena armena, Venise $\mathrm{r} 826$. - Les traductions du grec sont en partie produits de l'édition par R. Arnaldez, Cl. Mondésert et J. Pouilloux, Les œuvres de Philon d'Alexandrie, I sqq., Paris r96r sqq., en partie sont-ils les miennes.

2 Cf. H. A. Wolfson, Philo, and ed., rev., 2, Cambridge (Massachusetts) r948, p. 83-85.

s Cf. H. A. Wolfson, "The Philonic God of Revelation", The Harvard Theological Review, 53, r96o, p. 103. 


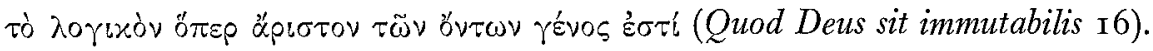
- Mais la connaissance complète et vraie de Dieu ne s'atteint que par révélation, dit Philon. Ainsi la révélation dont Philon se sert de mots divers (par exemple prophétie, vision, spectacle, extase, etc.) est une idée des plus importantes dans l'opinion religieuse de Philon.

Juif, Philon tient à la révélation au Sinaï comme un événement historique, mais en même temps il affirme que la révélation - le rencontre de l'homme avec Dieu - est continuée d'abord par les prophètes et puis par d'autres personnes qui par cette voie reçoivent des renseignements sur la volonté et la nature de Dieu. La révélation est donc une suite d'événements toujours en cours.

Mais comment se fait cette dernière forme de révélation et qu'est-qu'il implique? Qu'il implique une acquisation d'une certaine forme d'entente ou de connaissance est donné directement par la définition. Un bon point de départ pour la compréhension des descriptions de Philon concernant l'éxperience des révélations sera un des passages dans la vaste production de Philon qui concerne la révélation; il est raisonnable de choisir ici une des publications assez courtes comme point de départ, par exemple l'ouvrage intitulé De fuga et inventione.

Comme bien des fois Philon s'efforce de faire des réflexions philosophiques sur ce qu'impliquent certains termes de l'Ancient Testament. Un petit nombre de mots est tourné et retourné. Ainsi la première partie de l'ouvrage (De fuga et inventione $\mathrm{I}-\mathrm{I}$ 18) contient des explications sur ce qu'implique le mot purn' (fuga) et des expressions analogues, soit que ce soit la fuite de Hagar d'Abraham, la fuite de Jacob de Laban, la fuite de Jacob à Haran ou quelque chose d'analogue.

La seconde partie de la dissertation - c'est celle qui nous interesse en cette matière - De fuga et inventione II9-I75 s'agit d'un exposé systématique des questions relatives au mot eúpeoıs (inventio) et aux mots analogues. Le point de départ de Philon sont les mots dans la Genèse $16,7:$ Un

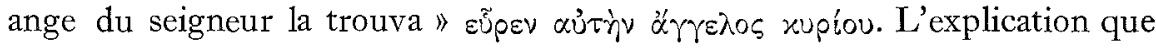
donne ensuite Philon peut être caractérisée justement comme systématique puisque Philon énumère avec exactitude et par ordre les possibilités qui sont attachées à l'action de chercher et l'action de trouver.

Philon dit d'abord que c'est utile que les idées du législateur sur l'action 
de trouver et l'action de chercher ne sont pas supprimées parce qu'il signale quelques-uns qui ne cherchent ni ne trouvent quelque chose et puis des autres qui font l'un et l'autre et ensuite quelques-uns qui n'ont fait qué ceci ou cela parmis lesquels il y en a qui ont cherché sans avoir trouvé et des autres qui ont trouvé bien qu'ils n'aient pas cherché (De fuga et inventione I20).

Dans la suite Philon examine les quatre catégories. Evidemment il y a peu de chose à dire sur la première catégorie : ceux qui ne cherchent ni ne trouvent quelque chose (De fuga et inventione II9-125). De la catégorie suivante, ceux qui cherchent et trouvent aussi, il est écrit qu'ils réfléchissent et considèrent et examinent tout avec soin. En outre il est écrit que celui qui s'exerce recueillera les fruits proportionnées à cela, c'est-à-dire la pénétration

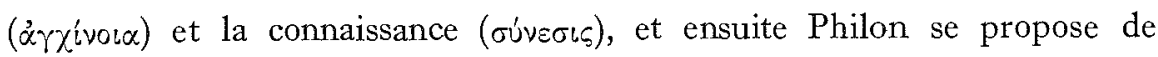
regarder mieux les hommes qui exercent et l'action de chercher et l'action de

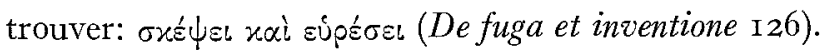

Ici dans l'exposé suivant il est évident que les expressions chercher et trouver chez Philon s'appliquent à plusieurs choses, mais que cela atteint le point culminant dans l'action de chercher Dieu. Car il est dit que l'action de chercher le meilleur de tout ce qui existe, la cause incomparable de toutes choses, cela nous porte de la joie dès l'instant que nous commençons notre recherche et cela ne nous laisse jamais sans fruit puisq'à cause de sa nature gracieuse il nous prévient avec ses grâces pures et chastes et se présente pour ceux qui cherchent à le voir non comme il est, ce qui est impossible puisque même Moïse détourna son regard parcequ'il avait peur de voir Dieu (cf. Exod. III, 6) mais autant que ce soit possible qu'un être tourne son regard versla puissance qui est hors de l'intelligence (De fuga et inventione $\mathrm{I} 4 \mathrm{I}$ ).

Pourtant Philon sait que ce n'est pas tout le monde qui réussit dans les aspirations de l'âme vers la connaissance. Cela est mentionné dans ce qu'ap-

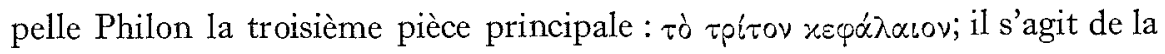
catégorie dans laquelle il y a bien de la recherche mais sans que l'action de

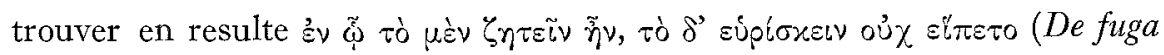
et inventione 143). Sur la recherche infructueuse de cette catégorie il est écrit chez Philon que comme les garçons et les hommes adultes n'aprennent pas les mêmes choses vu qu'il existe des sciences qui s'accordent avec chaque age, ainsi il y a des âmes qui sont créées simples même dans les corps qui sont en train d'arriver à la maturité et l'age adulte (De fuga et inventione I46). 
La quatrième et dernière catégorie comprend les gens qui trouvent sans chercher; la trouvaille se fait brusquement et avec précipitation sans efforts précédents pour chercher. Sur cela Philon dit qu'il y a des gens pour lesquels la trouvaille se plaît d'arriver précipitamment bien qu'il n'y ait pas

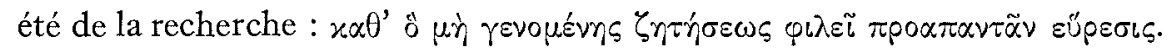
(De fuga et inventione I66). A cette catégorie appartient chaque sage qui ne tire sa sagesse d'aucun maitre; sur lui il est écrit qu'au moment de sa naissance la sagesse est mise à sa disposition descendue du ciel en haut et qu'il en boit des boissons pures et qu'il ne finit pas de s'enivrer de l'ivresse sobre qui consiste en la vraie connaissance (De fuga et inventione $\mathrm{I} 66$ ).

Ainsi, par l'exposition des quatres catégories différentes en lesquelles Philon divise les hommes selon leur possibilités de connaissance, nous avons obtenu les deux catégories qui nous interesse en cette matière, c'est-à-dire ceux qui cherchent et trouvent et ceux qui obtiennent la connaissance immédiatement sans chercher. Et avec la description de cette dernière catégorie nous sommes arrivés à ceux qui obtiennent l'expérience mystique de la divinité sans intermédiaire.

Un mot de passe pour la compréhension de l'expérience à laquelle Philon fait allusion ici est l'expression dont il se sert dans le passage déjà reféré de De fuga et inventione I66: s'enivrer avec l'ivresse sobre'. Ailleurs Philon traite l'expérience comme quelquechose qui arrive quand les âmes prennent une boisson forte "pour qu'elles se trouvent possédées d'une ivresse divine,

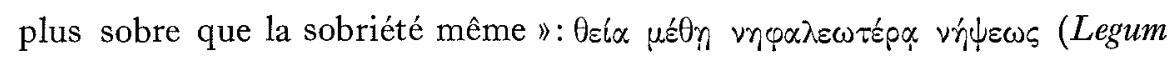
allegoriae III, 82). Aussi Philon dit de celui qui est enflammé de la reconnaissance à Dieu et le remercie avec une glorification « qu'il est ivre d'une

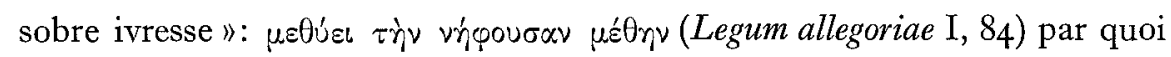
Philon soutient que la gratitude, c'est-à-dire l'ivresse sobre, lui est donnée par Dieu (cf. Legum Allegoriae I, 82).

Cette ivresse est accordée par Dieu². Les hommes peuvent bien travailler, faire des efforts et chercher et, comme on peut constater d'après certains passages chez Philon, ces efforts ont un certain mérite, mais il ne savent pas

${ }^{1}$ Cf. H. Lewy, Sobria Ebrietas, Untersuchungen zur Geschichte der antiken Mystik, Giessen I929.

${ }^{2}$ Cf. J. Daniélou, Philon d'Alexandrie, Paris I958, p. I96. 
produire l'expérience même de Dieu. Elle est accor dée comme l'ivresse sobre de la reconnaissance mentionnée ci-dessus.

L'expérience de Dieu arrive subitement puisque ce qu'il faut apprendre demande de la préparation pendant longtemps tandis que ce qui se fait grâce à la nature arrive vite et en dehors du temps ( $\alpha^{\prime} \chi \chi_{p o v o c}$ ) et de soi-même (De fuga et inventione 169 ). De soi-même veut dire en cette matière sans action humaine, seulement causé par Dieu. Philon dit que soit le commencement de la connaissance soit la semence de la perfection de cette connaissance depend de la nature qui est posée dans l'homme; mais selon Philon Dieu seul,

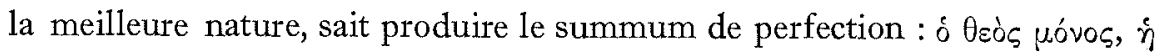

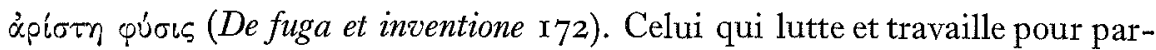
venir à la connaissance est plein d'inquiétude et de crainte, oui cela se voit de son teint pâle, dit Philon dans un autre traité: «celui qui en plus travaille, a la coleur de la pierre verte, car les amis de l'effort sont pâles par

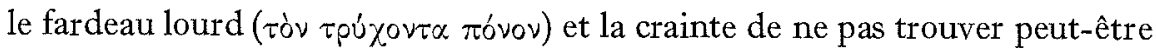
un fin conforme à leur prière " (Legum allegoriae $\mathrm{I}, 84$ ).

D'autre part Philon représente celui qui vit de l'instruction divine comme

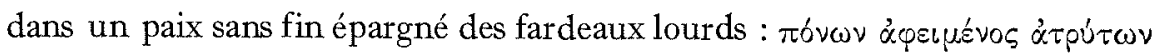
(De fuga et inventione $\mathrm{r} 73$ ). Il est interessant de remarquer que Philon se sert exactement des mêmes mots pour désigner les fardeaux lourds de l'ambitieux dans deux traités différents. La paix sans fin qui entoura celui qui viva de l'instruction divine peut être decrit par Philon comme une source surgeant subitement dans l'âme; dans De fuga et inventione $x_{37}$ Philon dit qu'il coule du mot divin un flot interminable de toute sorte d'instructions et d'enseignements quand Dieu satisfait le chercheur; dans De migratione Abrahami 30 la question est mentionnée un peu différemment, mais la symbole de la sorce est presque la même : «la source d'où les biens coulent à flots, c'est la com-

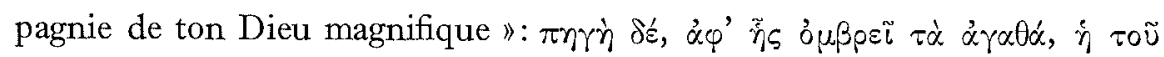

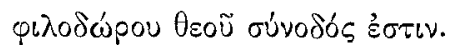

Après avoir donné un exposé des points de vue de Philon comme ils paraissent dans notre point de départ, De fuga et inventione, nous pouvons nous occuper avec avantage d'autres parties des oeuvres de Philon pour examiner des éléments dans les descriptions de l'expérience mystique de Dieu chez Philon.

Dans un exposé fort personel Philcon raconte un peu plus loin dans le 
traité susmentionné : De migratione Abrahami le suivant : "Je ne crains pas de dire ici une expérience personelle, qui m'est familière pour s'être vingt fois ${ }^{1}$ répétée. J'avais décidé certains jours de suivre la redaction ordinaire des traités philosophiques : je voyais exactement les éléments de la composition, mais je rencontrais qu'une pensée impuissante et stérile; sans ressource, j'ai abandonné la partie, en stigmatisant la présomption de la pensée, mais tout impressionné par la vigueur de l'Être dont il dépendait que le sein de l'âme soit ouvert et fermé. Au contraire, j'étais venu, certains jours, comme vide : je fus tout d'un coup rempli de ses idées répandues comme neige et invisiblement semées d'en haut, au point de passer en extase sous l'effet de la possession divine, de ne plus rien reconnaître, ni le lieu où j'étais ni les témoins, ni moi-même, ni ce qu'on pouvait dire ou écrire sur le sujet. La raison en est que Dieu fait pour ainsi dire saisir l'invention des idées comme le bénefice tiré de la lumière du jour : une vue perçante, un éclat merveilleux de transparence, tel que les yeux peuvent en jouir devant des objets que leur clarté fait voir parfaitement " (De migratione Abrahami 34-35).

Il serait tentant d'interpréter cet exposé comme seulement une description de la peine d'un auteur d'écrire et sa disposition suivante. Cependant en soi plusieurs expressions dans l'exposé de Philon sont trop forts pour une telle interprétation. En somme on pourrait l'appeler une description des situations avec une inspiration absente et des situations où l'inspiration vient, mais puis il faut se rappeler le sens de l'inspiration comme "insufflation》, dans ce cas insufflation de quelque chose de divine. Bien qu'on l'interpréte ainsi c'est pour Philon aussi une partie de la révélation divine, puisque chacun qui interpréte la loi (comme Philon aussi l'a regardé comme sa mission essentielle) selon Philon ne savait l'interpréter que par la participation à la révélation continuée de la nature de Dieu et en même temps de sa volonté.

L'expérience mystique peut paraitre comme quelque chose d'extérieur dans les descriptions de Philon. Quand l'âme a l'expérience mystique cela se ressent comme une joie qui peut être interprétée comme une sorte de folie. Entre autres choses c'est decrit dans De ebrietate. Ici on lit que «l'âme qui est comblée de cette grâce aussitôt est joyeuse, sourit et danse. Elle est

${ }^{1}$ Sic la traduction par J. Cazeaux, dans l'édition par R. Arnaldez, Cl. Mondésert et

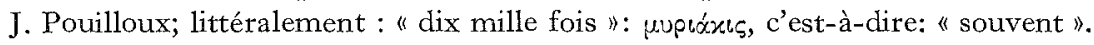


transportée d'enthousiasme ( $\beta \varepsilon \beta \dot{\alpha} \alpha \chi \varepsilon \cup \tau \alpha \alpha)$, au point de paraître, aux yeux

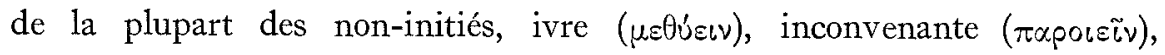
hors d'elles-mêmes ( $\left.\xi_{\xi} \xi \sigma \tau \dot{\alpha} v \alpha \iota\right)$ ) (De ebrietate $\left.{ }^{4} 46\right)$. Selon Philon cette expérience peut agir sur le corps; comme si souvent chez Philon des observations physiologiques se combinent avec des observations philosophiques comme nous l'avons déjà constaté par la mention de Philon du teint pâle des ambitieux. Aussi il est écrit chez Philon dans De ebrietate: «Car chez ceux qui sont possedés de Dieu ( $\theta \varepsilon \circ$ opopítors) non seulement l'âme est générale-

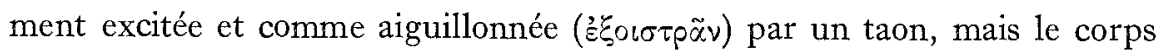
est empourpré et embrasé par le flot débordant et rechauffant d'une joie intérieure qui repand à l'extérieur ce qu'elle éprouve. C'est pourquoi beaucoup de fous sont trompés et soupçonnent des hommes sobres d'être pris

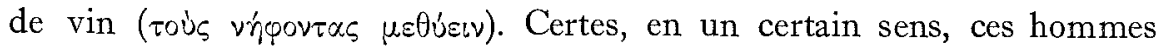

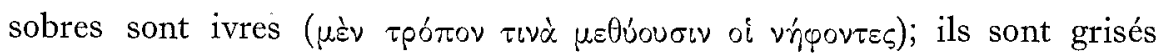
comme d'un vin pur de leurs biens accumulés et reçoivent de la parfaite vertu la coupe de l'amitié " (De ebrietate 147-148).

Une foi l'expérience de Dieu obtenue, on le ressent selon Philon comme une chose qu'il faut ne pas reperdre. Autant est grand le désir de parvenir à l'expérience mystique autant est grande la crainte que cette expérience mystique finisse et que l'âme rentre dans son état précédent de vide. Cela est écrit chez Philon entre autres choses dans son Questiones et solutiones in Genesin seulement conservé en arménien où Philon dit $(\mathrm{IV}, 4)$ dans un commentaire à la Genèse XVIII, 3 sur la prière d'Abraham que c'est quelque chose de grand dont il prie Dieu, c'est-à-dire que Dieu ne le depasse ou ne s'éloigne en laissant son âme deserte et vide. Car la limite de la béatitude c'est la présence de Dieu qui comble l'âme de toute sa lumière spirituelle et éternelle, et la limite du mal c'est la disparition, car immédiatement après les ténébres lourds et profonds prennent le dessus.

Celui duquel Dieu est absent est plein de chagrin et souffre, Philon affirme plus loin dans Questiones et solutiones in Genesin (IV, I8); la présence de Dieu signifie que l'homme est comblé de joie. Cette joie est défini par Philon ibidem comme apparemment consistant en la réception des lumières des plus rayonnantes d'en haut et si Dieu se retourne à l'âme et l'âme se retourne vers lui il le fait aussitôt se remplir de joie, est-il écrit dans le texte arménien. 
Les relations entre Dieu et celui qui le rencontre immédiatement dans l'expérience mystique peuvent être exprimées différement chez Philon. Parlant des relations de Moïse avec Dieu Philon mentionne la présence ( $\sigma u ́ v o \delta o s)$ du Dieu unique chez Moïse (De fuga et inventione I40). On peut se servir d'autres expréssions aussi, comme par exemple dans $D e$ posteritate Caini 92 où il est écrit que celui qui voit Dieu attiré à lui par la beauté imposante est venu à lui qu'on voyait comme lot et part.

C'est une question combien d'expérience le mystique sait tirer de la divinité. Cela n'est pas toujours exprimé tout net et logiquement par Philon. Comme nous venons de comprendre par la citation de De posteritate Caini

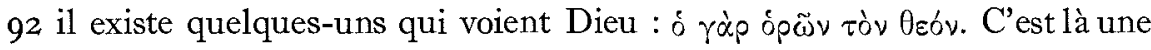
question de savoir combien le mot ópä̀ implique. De même ailleurs chez Philon, par exemple De fuga et inventione I65, on est prévenu contre des tentatives de voit le Très-Haut. Dans De specialibus legibus I, 44 il est écrit dans l'interprétation de Philon des paroles de Dieu à Moïse que sa nature réelle ne peut être aperçue par aucun être; Dieu aime bien, lit-on, offrir tout ce qui s'accorde avec celui qui reçoit, mais l'homme n'est pas capable de percevoir tout ce que Dieu pourrait offrir sans peine; tout ce qu'il peut recevoir on le lui offrira, mais l'action de comprendre ( $(\alpha \tau \alpha \dot{\lambda} \lambda \eta \psi \psi(s)$ Dieu c'est plus qu'une nature humaine, même le monde entier ne pourra contenir. Plus loin il est dit dans De specialibus legibus I, 49 que Moïse ne doit pas espérer de jamais le comprendre $(x \alpha \tau \alpha \lambda \alpha \beta \beta \tilde{\varepsilon} \nu)$, lui ou ses puissances con-

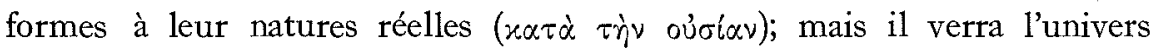
avec tout ce qu'il renferme non avec l'oeil du corps mais avec l'oeil de l'âme.

Ici il sera raisonnable de réfléchir si le verbe $\varkappa \alpha \tau \alpha \lambda \alpha \mu \beta \alpha \dot{v} \varepsilon \iota \nu$ a joué un rôle plus fort chez Philon que le verbe ópäv. Selon toute probabilité on pourrait impliquer des expréssions comme Quod Deus sit immutabilis 79-8I d'où il ressort que le connaissable de Dieu a été modéré pour que les hommes ne périssent en connaissant. Il ne s'agit guère ici que d'une connaissance de l'existence de Dieu mais aussi d'une certaine forme de connaissance de sa nature réelle, quoique modérée. 